\title{
Monitoring Ponatinib in a Child with Philadelphia Chromosome-Positive Acute Lymphoblastic Leukemia
}

\author{
Kazuki Tanimura ${ }^{a}$ Kai Yamasaki $^{a}$ Yuki Okuhiro $^{a}$ Kota Hira $^{a}$ \\ Chika Nitani $^{a}$ Keiko Okada $^{a}$ Hiroyuki Fujisaki $^{a}$ Kana Matsumoto ${ }^{b}$ \\ Junichi Hara ${ }^{a}$ \\ aDepartment of Pediatric Hematology and Oncology, Children's Medical Center, Osaka \\ City General Hospital, Osaka, Japan; ${ }^{b}$ Department of Clinical Pharmaceutics, Faculty of \\ Pharmaceutical Sciences, Doshisha Women's College of Liberal Arts, Kyoto, Japan
}

\section{Keywords}

Philadelphia chromosome-positive acute lymphoblastic leukemia · Ponatinib · Plasma concentration

\begin{abstract}
Ponatinib is a third-generation tyrosine kinase inhibitor (TKI) reported to show a higher efficacy for adult Philadel phia chromosome-positive acute lymphoblastic leukemia ( $\mathrm{Ph}+\mathrm{ALL}$ ) than other TKIs. However, few studies describe ponatinib for pediatric Ph+ALL; therefore, the efficacy, safety, and optimal dosage have not been determined. Here, we report a 3-year-old girl with $\mathrm{Ph}+\mathrm{ALL}$ treated by a ponatinib-containing regimen with therapeutic drug monitoring in the plasma and cerebrospinal fluid (CSF). In our case, a ponatinib-containing regimen was able to keep minimal residual disease negative, and the pharmacokinetics (PKs) of plasma ponatinib resembled that previously reported in adults. Penetration to the CSF was extremely limited. Thus, ponatinib was feasible and effective for a child with $\mathrm{Ph}+\mathrm{ALL}$, although the plasma concentration of ponatinib varied significantly throughout the treatment. The appropriate dosage should be confirmed in a prospective trial, including a detailed PK study.
\end{abstract}

(c) 2021 The Author(s).

Published by S. Karger AG, Basel

\section{Introduction}

The clinical outcome of pediatric Philadelphia chromosome-positive acute lymphoblastic leukemia (Ph+ALL) has improved dramatically since the introduction of imatinib. Imatinib has enabled most children with $\mathrm{Ph}+\mathrm{ALL}$ to avoid allogenic hematopoietic stem 


\section{Case Reports in Oncology}

\begin{tabular}{|c|c|}
\hline Case Rep Oncol 2021;14:2 & \\
\hline DOI: 10.1159/000511071 & $\begin{array}{l}\text { (c) } 2021 \text { The Author(s). Published by S. Karger AG, Base } \\
\text { www.karger.com/cro }\end{array}$ \\
\hline
\end{tabular}

Fig. 1. A Pharmacokinetics (PKs) of ponatinib after initiation (dose: $10 \mathrm{mg} /$ day) during re-induction chemotherapy. B PK of ponatinib during steady phase (solid line: $10 \mathrm{mg} /$ day, dotted line: $15 \mathrm{mg} /$ day). The steady-phase plasma concentration was measured after the patient received the same dose for a week. C Schematic of ponatinib trough plasma concentration during chemotherapy. Intensive chemotherapy included re-induction block 2 and intensification block 2. Daily ponatinib dose was adjusted between 0 and $15 \mathrm{mg}$ based on trough plasma concentration and medical adverse events.
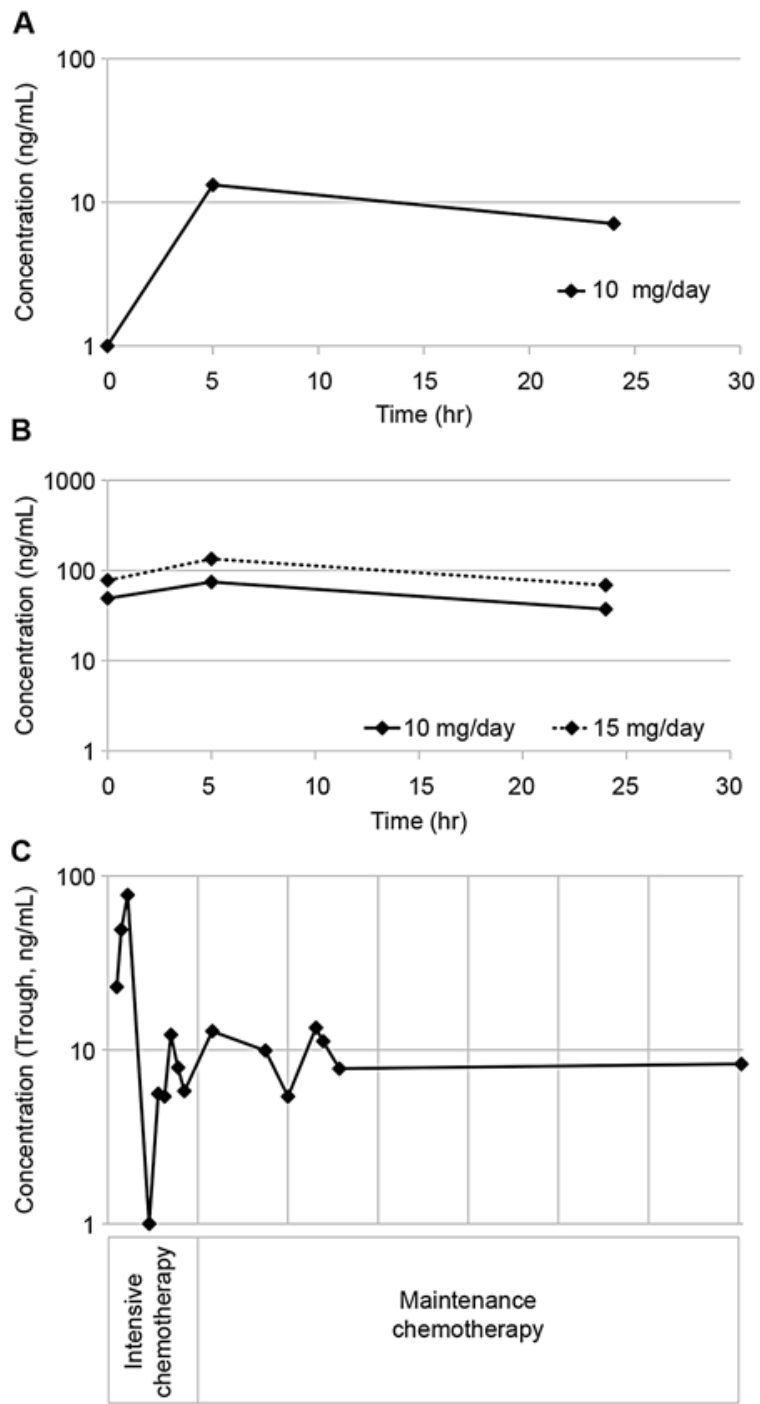

cell transplantation (allo-HSCT) as the first complete response [1]. However, dasatinib, a second-generation tyrosine kinase inhibitor (TKI), failed to show a higher efficacy than imatinib in the Children's Oncology Group (COG) AALL0622 trial [2]. Ponatinib (ICLUSIG ${ }^{\circledR}$ ) is a third-generation TKI reported to show higher efficacy for adult Ph+ALL than other TKIs [3]. In adult Ph+ALL, allo-HSCT for the first complete response is still a standard treatment. However, some clinical studies have suggested the possibility of avoiding alloHSCT by using ponatinib-containing regimens [3]. However, few studies describe ponatinib for pediatric Ph+ALL; therefore, the efficacy, safety, and optimal dosage have not been determined.

Here, we report a case of pediatric $\mathrm{Ph}+\mathrm{ALL}$ treated with a ponatinib-containing regimen with therapeutic drug monitoring in the plasma and cerebrospinal fluid (CSF). Ponatinib concentrations in the plasma and CSF were measured using liquid chromatography-tandem mass spectrometry as previously described, with some modifications [4]. 
Tanimura et al.: Monitoring Ponatinib in a Ph+ALL Child

\section{Case Report}

A 3-year-old girl experienced long-lasting fever and pallor and was admitted to our hospital. She presented with systemic lymphadenopathy and hepatosplenomegaly. Laboratory analysis showed the following: white blood cell count: 595,720/ $\mu \mathrm{L}$ (blasts: $49.5 \%$ ), hemoglobin: $3.6 \mathrm{~g} / \mathrm{dL}$, and platelets: $29,000 / \mu \mathrm{L}$. There were no extramedullary lesions; however, CSF cytology indicated central nervous system (CNS) infiltration. Minor BCR-ABL transcripts were detected by fluorescent in situ hybridization and quantitative reverse transcription-polymerase chain reaction (RT-PCR) $\left(6.7 \times 10^{4}\right.$ copies/ $\mu$ g RNA). She responded poorly to prednisolone. Therefore, we started induction chemotherapy based on the Japan Association of Childhood Leukemia Study (JACLS) HR-02 concurrent with dasatinib $60 \mathrm{mg} /$ $\mathrm{m}^{2} /$ day [5]. Her parents provided consent for treatment. After induction chemotherapy, complete cytogenetic remission was achieved; however, minimal residual disease (MRD) measured by RT-PCR of minor BCR-ABL fusion transcripts was detected at a level of $1 \times 10^{-4}$. Dasatinib was continued based on the COG AALL0622 protocol. After intensification block 1 , MRD was still detected. Therefore, after obtaining written informed consent, we switched dasatinib to ponatinib from re-induction block 2 .

Initially, we started ponatinib at a dose of $10 \mathrm{mg} /$ day (equivalent to $30 \mathrm{mg} /$ day in adults by Augsberger's method). The daily ponatinib dose was adjusted by assessing the plasma concentration and adverse clinical events. The plasma concentration-time profiles following a single oral dose and during steady-state are shown in Figure 1A and B. In addition, penetration to the CSF was extremely limited with a peak concentration of $0.1 \mathrm{ng} / \mathrm{mL}$. After increasing to $15 \mathrm{mg} /$ day, the patient developed hypertension and required an antihypertensive agent. After high-dose methotrexate therapy, we needed to stop ponatinib because of acute kidney injury owing to delayed methotrexate elimination. After 3 weeks of withdrawal, ponatinib was restarted at a dose of $5 \mathrm{mg} /$ day and gradually increased according to plasma concentration. After the introduction of ponatinib, MRD disappeared after re-induction block 2. Therefore, ponatinib was continued concurrently with the AALL0622 protocol. Finally, ponatinib at a dose of $5 \mathrm{mg}$ was continued throughout maintenance chemotherapy. During the maintenance phase, she presented several mild complications, such as abdominal pain, otalgia, aspartate aminotransferase and alanine aminotransferase elevation, and hyperbilirubinemia; however, these complications were controllable by standard supportive therapy. Growth deceleration in height was observed throughout her treatment. We evaluated the plasma ponatinib concentration non-periodically during her treatment (Fig. 1C). During the maintenance phase, the plasma trough concentrations of ponatinib at a dose of $5 \mathrm{mg} /$ day were lower than those during the re-induction phase. At present, she has completed maintenance chemotherapy and receives careful observation without HSCT.

\section{Discussion}

This is the first report of the detailed monitoring of ponatinib in a pediatric patient with $\mathrm{Ph}+\mathrm{ALL}$. The pharmacokinetics (PKs) during the initiation phase resembled those in a phase I study of adult Ph+ALL [3], but the trough value of ponatinib varied significantly throughout treatment. Following single $45-\mathrm{mg}$ doses of ponatinib in the adult, peak concentrations were observed approximately $4 \mathrm{~h}$ after administration, and the terminal elimination half-life was $22 \mathrm{~h}$. In adults, steady-state conditions are typically achieved within 1 week of continuous dosing. Moreover, it has been reported that the trough value of ponatinib is stable throughout each treatment phase in adult Ph+ALL cases [6]. In our case, the trough value of ponatinib varied throughout each treatment phase despite the same daily dose, which implied insta-

\section{Karger'}


bility of the ponatinib plasma concentration. One possible reason is the effect of chemotherapeutic agents on metabolism. Ponatinib is metabolized to an inactive carboxylic acid by esterases, amidases, and CYP3A4, CYP2C8, CYP2D6, and CYP3A5 to an N-desmethyl metabolite that is four times less active than ponatinib. The carboxylic acid and $\mathrm{N}$-desmethyl metabolite comprise 58 and $2 \%$ of the circulating ponatinib, respectively. The metabolic activity of CYP enzymes is easily affected by many antileukemic agents, such as vincristine, cyclophosphamide, and etoposide. As the pediatric ALL regimen is more complicated than the adult ALL regimen, the instability of the ponatinib plasma concentration in pediatric cases may be different from that in adult cases.

Severe adverse events associated with a high ponatinib concentration have been reported, and even concentrations as low as $40 \mathrm{~nm}$ can induce severe adverse events [6]. Therefore, it is important to determine the appropriate dose of ponatinib based on the trough concentration in pediatric cases. The CSF penetration of ponatinib was as low as that reported previously [3]. The efficacy of ponatinib for CNS leukemia has not yet been proven. It has been reported that dasatinib slightly crosses the blood-brain barrier (BBB) and is an efficient therapy for CNS Ph+ALL [7]. However, there are limited data on whether ponatinib crosses the BBB as well. Abid and De Mel [8] presented a case that showed CNS relapse during ponatinib treatment and concluded that ponatinib does not penetrate the CSF. However, Masuda et al. [9] showed successful experiences for Ph+ALL cases with CNS relapse using ponatinib concomitant with ALL chemotherapy. From our result, ponatinib showed a slight CNS penetration. Additionally, our case is now successfully managed without cranial irradiation, though CNS infiltration was positive. Therefore, our case might demonstrate the potential utility of ponatinib for managing CNS leukemias.

\section{Conclusion}

Ponatinib was feasible and effective for a child with $\mathrm{Ph}+\mathrm{ALL}$. The plasma concentration of ponatinib varied significantly throughout treatment. The appropriate dosage may need to be determined by considering simultaneously used anti-leukemic agents. Further clinical trials, including a detailed PK study, are required.

\section{Acknowledgements}

We thank our colleagues and the medical staff at the Osaka City General Hospital who helped with the treatment of this child.

\section{Statement of Ethics}

This case report complied with the guidelines for human studies and included evidence that the research was conducted ethically in accordance with the World Medical Association Declaration of Helsinki. Written informed consent was obtained from the patient for publication of this case report and any accompanying images.

\section{Conflict of Interest Statement}

The authors declare no conflict of interest.

\section{Karger'}




\section{Case Reports in Oncology}

Case Rep Oncol 2021;14:24-28

\begin{tabular}{l|l}
\hline DOI: $10.1159 / 000511071$ & ○ 2021 The Author(s). Published by S. Karger AG, Basel
\end{tabular} www.karger.com/cro

Tanimura et al.: Monitoring Ponatinib in a Ph+ALL Child

\section{Funding Sources}

Our work was not funded from any sources.

\section{Author Contributions}

K.T. wrote the manuscript and K.Y. intensively revised it. K.T. and K.Y. treated the patient and collected and interpreted the data. K.M. measured the plasma and CSF concentration of ponatinib. Y.O., K.H., C.N., K.O., H.F., and J.H. supervised the entire study process. All authors have read and approved the final manuscript.

\section{References}

1 Schultz KR, Bowman WP, Aledo A, Slayton WB, Sather H, Devidas M, et al. Improved early event-free survival with imatinib in Philadelphia chromosome - Positive acute lymphoblastic leukemia: A Children's Oncology Group Study. J Clin Oncol. 2009 Nov;27(31):5175-81.

2 Slayton WB, Schultz KR, Kairalla JA, Devidas M, Mi X, Pulsipher MA, et al. Dasatinib plus intensive chemotherapy in children, adolescents, and young adults with Philadelphia chromosome-positive acute lymphoblastic leukemia: Results of Children's Oncology Group Trial AALL0622. J Clin Oncol. 2018 Aug;36(22):230613.

3 Tojo A, Kyo T, Yamamoto K, Nakamae H, Takahashi N, Kobayashi Y, et al. Ponatinib in Japanese patients with Philadelphia chromosome-positive leukemia, a phase 1/2 study. Int J Hematol. 2017 Sep;106(3):385-97.

4 Andriamanana I, Gana I, Duretz B, Hulin A. Simultaneous analysis of anticancer agents bortezomib, imatinib, nilotinib, dasatinib, erlotinib, lapatinib, sorafenib, sunitinib and vandetanib in human plasma using LC/MS/ MS. J Chromatogr B Analyt Technol Biomed Life Sci. 2013 May;926:83-91.

5 Hasegawa D, Imamura T, Yumura-Yagi K, Takahashi Y, Usami I, Suenobu SI, et al. Risk-adjusted therapy for pediatric non-T cell ALL improves outcomes for standard risk patients: results of JACLS ALL-02. Blood Cancer J. 2020 Feb;10(2):23.

6 Cortes JE, Kantarjian H, Shah NP, Bixby D, Mauro MJ, Flinn I, et al. Ponatinib in refractory Philadelphia chromosome-positive leukemias. N Engl J Med. 2012 Nov;367(22):2075-88.

7 Marchesi F, Salvatorelli E, Renzi D, Mengarelli A, Minotti G, Menna P. Efficacy and safety of low dose ponatinib in a case of Ph-positive acute lymphoblastic leukaemia. Br J Haematol. 2019 Aug;187(1):e15-7.

8 Abid MB, De Mel S. Does ponatinib cross the blood-brain barrier? Br J Haematol. 2017 Nov;179(3):497-8.

9 Masuda K, Nakazato T, Nishiyama-Fujita Y, Ito C, Ogura S, Mizuno K, et al. Successful treatment with ponatinib for central nervous system relapse of Philadelphia chromosome-positive B-cell acute lymphoblasticleukaemia. Intern Med J. 2019 0ct;49(10):1332-4. 